acute respiratory exacerbations in children with cystic fibrosis? J Pediatr 1980;97:144-7.

2 Westphal O. Bacterial endotoxins. Int Arch Allergy Appl Immunol 1975;49:1-43.

3 Taylor G, Slade M, Stern C, Silverman M. A new micro-method of endotoxin detection. J Appl Bacteriol $1981 ; 51$ :XX.

4 Webster CJ. Principles of a quantitative assay for bacterial endotoxins in blood that uses limulus lysate and a chromogenic substrate. J Clin Microbiol 1980;12:644-50.
${ }^{5}$ C 4 ridis D, Reinhold RB, Woodruff PWH, Fine J. Endotoxaemia in man. Lancet $1972 ; \mathrm{i}: 1381-6$.

6 Prytz H, Holtst-Christensen J, Korner B, Liehr H. Portal venous and systemic endotoxaemia in patients withou liver disease and systemic endotoxaemia in patients with cirrhosis. Scand J Gastroenterol 1976;11 :857-63.

Correspondence to Dr M Silverman, Royal Postgraduate School of Medicine, Hammersmith Hospital, Ducane Road, London W12 0HS.

Received 11 July 1983

\title{
Three successfully treated cases of nonamoebic liver abscess
}

\author{
P K H TAM, H SAING, AND J T K LAU \\ Division of Paediatric Surgery, Department of Surgery, University of Hong Kong
}

SUMMARY Three previously healthy children developed nonamoebic liver abscess. Diagnosis was aided by liver scan, ultrasound assessment, and computed tomography. A variety of surgical procedures were successful in achieving proper drainage, resection of chronic lesions, and resolution of associated cholangitis.

Nonamoebic liver abscess in children is an uncommon occurrence and only about 100 cases have so far been reported. ${ }^{1-4}$ Most of these cases occurred in compromised hosts with either underlying malignancy, septicaemia, or chronic granulomatous disease and only about $20 \%$ in previously healthy children. ${ }^{1}$ Mortality is high-a figure of $34 \%$ was reported by Cushid ${ }^{1}$ in his review of the published reports. In the past 10 years we have treated three uncompromised children with nonamoebic liver abscesses with satisfactory outcome; each patient requiring a different surgical approach. The need for awareness of this condition and individual management is stressed.

\section{Case reports}

Case 1. An 18 month old boy presented with fever of $1 \frac{1}{2}$ months duration. He had no abdominal pain; there was right upper quadrant tenderness but the liver was not palpable. Blood and liver function tests were all normal, but his chest radiograph showed elevation of the right hemidiaphragm and a liver scan showed a lesion in the right lobe.

At laparotomy an abscess was confirmed; the rest of the liver being studded with small chronic septic nodules. The common bile duct was dilated and packed with 14 ascaris and purulent bile. These were removed and the common duct and abscess drained separately. Escherichia coli was found in both pus and bile. He made an uneventful recovery after a course of antibiotics and antihelminths, and at follow up 8 years later his cholangiogram and liver scan were normal.

Case 2. A 9 year old boy was referred after 6 months of swinging fever, chills, and rigors. He had had two mild attacks of abdominal pain not requiring treatment. Examination showed a tender enlarged liver. Initial investigations in another hospital showed a mild anaemia, raised erythrocyte sedimentation rate $(85 \mathrm{~mm}$ in the first hour), alkaline phosphatase $(358 \mu \mathrm{mol} / 1(50 \mathrm{KA} \mathrm{U} / 100 \mathrm{ml}))$, serum aspartate transaminase $(100 \mu \mathrm{mol} / \mathrm{l}(208 \mathrm{Rf} \mathrm{U} / \mathrm{ml}))$, serum alanine transaminase $(80 \mu \mathrm{mol} / 1$ (167 $\mathrm{Rf}$ $\mathrm{U} / \mathrm{ml})$ ). Leucocyte count, bilirubin, albumin, blood culture, and an ultrasound scan of the liver were all normal. After prolonged empirical antibiotic treatment failed he was referred to this hospital where we repeated ultrasound and found a $4 \mathrm{~cm}$ lesion in the right lobe. A liver scan showed similar results.

At laparotomy there were multiple old abscesses throughout the liver, with three considerably larger lesions-two on the right and one on the leftmeasuring $6 \mathrm{~cm}, 5 \mathrm{~cm}$, and $3 \mathrm{~cm}$ at their largest diameter. All were necrotic granulomata and mainly solid. Wedge excisions of the larger granulomata were performed. Cultures were all sterile. The boy developed a subphrenic haematoma requiring evacuation and a right pleural effusion but recovered well after a three week course of antibiotics. Two and a half years later he was well and had a normal liver scan.

Case 3. A 12 year old girl complained of fever, chills, and rigors for one month but had no abdominal pain. Examination showed a palpable liver edge and right upper quadrant tenderness. She had leucocytosis $\left(16 \times 10^{9} / 1\right) ; 16000 / \mathrm{mm}^{3}$ and a raised alkaline phosphatase $(361 \mu \mathrm{mol} / 1$ (51 KA U/100 ml)) but other parameters of liver function were all normal. Liver scan showed a solitary $5 \mathrm{~cm}$ lesion in right lobe. Computed tomography showed multiple low 


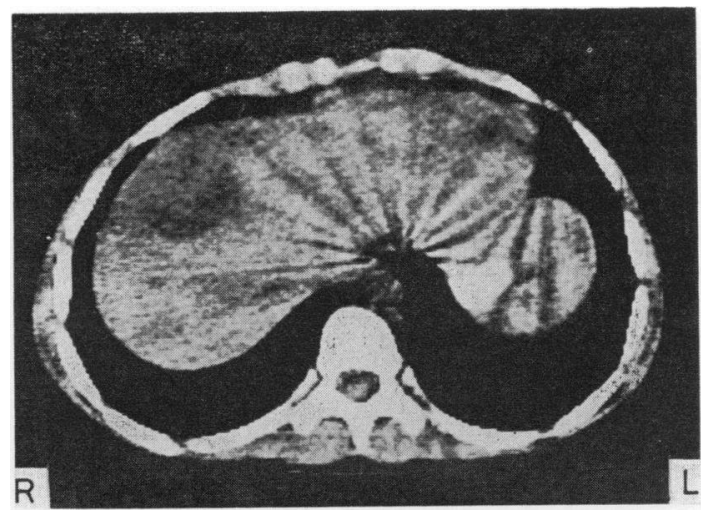

Figure Computed tomogram showing a solitary lesion in the right lobe of liver.

density lesions of various sizes in the right lobe, with one larger lesion (Figure).

At laparotomy two abscesses deep in the right lobe were found, and each drained $20 \mathrm{cc}$ of pus. Staphylococcus aureus was isolated in the pus and appropriate antibiotics were given. Apart from developing a sterile right pleural effusion, her recovery was smooth.

\section{Discussion}

Liver abscess in children remains a difficult diagnostic problem owing to both its rarity and its non-specific clinical features. As late as 1977 reports still indicated that the diagnosis in over $60 \%$ of cases was not made until necropsy, despite the presence of fever and hepatomegaly. ${ }^{1}$ In our experience only prolonged unexplained fever is a consistent symptom, and even abdominal pain may be absent or minimal. Right upper quadrant tenderness is another clinical clue. Blood tests indicating leucocytosis and altered liver function are helpful when positive but are more commonly negative even if there is extensive hepatic sepsis. Isotope liver scan has been most commonly used for diagnosis but is being increasingly criticised for its low sensitivity. Ultrasound assessment represents an improvement but its accuracy depends on interpretative skills, as indicated by our second patient whose first scan was reported as normal. Computed tomography promises to be more sensitive ${ }^{5}$ and our third patient may represent the first documented application in diagnosis of liver abscess in children.

The management of nonamoebic liver abscess is surgical and the approach should be individual. As in our first patient, cholangitis may be an underlying cause, necessitating common duct exploration for satisfactory eradication of the disease. Chronic reactive changes after prolonged antibiotics may render the lesions unresponsive to simple drainage, requiring resection as in our second child. Finally, there is no substitute for laparotomy and careful palpation for detection of liver abscess, as even ultrasound and computed tomography may not be able to show all important lesions.

\section{References}

1 Cushid MJ. Pyogenic hepatic abscess in infancy and childhood. Pediatrics 1978;62:554-9.

2 Larsen LR, Raffensperger J. Liver abscess. J Pediatr Surg 1979;14:329-31.

3 Harrington E, Bleicher MA. Cryptogenic hepatic abscess in two uncompromised children. J Pediatr Surg 1980;15: 660-2.

4 Arya LS, Ghani R, Abdali S, Singh M. Pyogenic liver abscesses in children. Clin Pediatr (Phila) 1982;21:89-93.

5 Buchman TG, Zuidema GD. The role of computerized tomographic scanning in the surgical management of pyogenic hepatic abscess. Surg Gynecol Obstet 1981 ;153: $1-9$.

Correspondence to Dr P K H Tam, Division of Paediatric Surgery, Department of Surgery, University of Hong Kong, Queen Mary Hospital, Hong Kong.

Received 15 June 1983

\title{
Selective placement of bronchial suction catheters in intubated neonates
}

\author{
M PLACZEK AND M SILVERMAN
}

Department of Paediatrics and Neonatal Medicine, Royal Postgraduate Medical School, Hammersmith Hospital, London

SUMMARY Flexible suction catheters were passed through the endotracheal tubes of infants undergoing mechanical ventilation, just before chest radiographic examination for clinical purposes. With the head straight, 7 of 10 straight catheters entered the right main bronchus but with the head turned, 17 of 20 straight catheters and 19 of 20 curved tip catheters entered the contralateral bronchus.
Mechanical ventilation of newborn babies is not infrequently accompanied by pulmonary atelectasis related to mechanical ventilation, 12 or tracheal suction, ${ }^{3}$ or after extubation. ${ }^{2}$ It occurs more commonly in low birthweight infants ${ }^{1}$ and after prolonged periods of intubation. ${ }^{2}$ The management of lobar atelectasis requires prompt recognition of the problem, suctioning of the tracheobronchial tree, and reinflation of the affected lung. 